Europhys. Lett., 23 (6), pp. 401-407 (1993)

\title{
Dynamics of a Secondary Instability in Bénard-Marangoni Convection with Unidimensional Heating.
}

\author{
J. Burguete, H. L. Mancini $\left({ }^{*}\right)$ and C. Pérez-García (**) \\ Departamento de Física y Matemática Aplicada, Facultad de Ciencias \\ Universidad de Navarra - E-31080 Pamplona, Navarra, Spain
}

(received 3 March 1993; accepted in final form 29 June 1933)

PACS. 47.20 - Hydrodynamic stability and instability.

PACS. 47.35 - Waves.

\begin{abstract}
The dynamics of Bénard-Marangoni convection with unidimensional heating in a pure fluid is studied experimentally. Convection begins with rolls parallel to the heater. The characteristics of these primary rolls have been determined. When the temperature difference across the liquid layer is increased beyond a critical value a secondary instability appears. Motions transverse to the heater with a definite wavelength can be seen. Moreover, for small angles between the heater and the fluid surface, the pattern drifts along the heater with a velocity that depends almost linearly on the inclination. A phenomenological phase equation is proposed to interpret this observation.
\end{abstract}

Convective motions due to a quasi-one-dimensional (1D) heating have been studied in different contexts. Kayser and Berg [1] studied the surface deformation of a fluid layer heated by a resistive wire on the bottom of a convective cell. The main result of this experiment is that the free surface just above the wire deflects to a concave or convex surface, depending on the liquid depth. This effect can be explained as a competition between temperature-depending surface tension forces that lead to a concave free surface (Bénard effect) and the buoyancy forces that tend to make the surface convex (Rayleigh effect). Later on, Anthore et al. [2] noticed that a laser beam that crosses the fluid in a small convective cell parallel to the free liquid surface begins to heat. These authors realized that when the laser beam crosses the fluid near the free liquid surface the beating has the same period as the surface deformation. The same group carried out a different experiment to elucidate the nature of those oscillations [3]. In the former studies they placed a short $(L=3 \mathrm{~cm})$ resistive wire just below the free surface and they determined the temperature thresholds for the oscillatory instability of the free surface due to a Bénard effect.

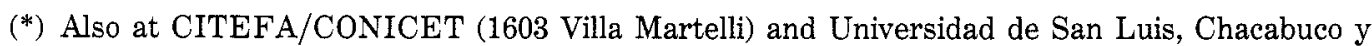
Pedernera, 5700 San Luis, Argentina.

(**) Also at Departament de Física Fonamental, Facultat de Física, Universitat de Barcelona, Av. Diagonal 647, 08028 Barcelona, Catalonia, Spain. 
TABLE I. - Thermal conductivities of the cell.

\begin{tabular}{lcccccc}
\hline & Air & Plexiglas & Glass & Rubber & Copper & Silicon oil \\
\hline $\begin{array}{l}\text { density } \\
\left(\mathrm{kg} / \mathrm{m}^{3}\right)\end{array}$ & 1.662 & 920 & 2600 & 1000 & 8920 & 910 \\
$\begin{array}{l}\text { heat capacity } \\
\begin{array}{l}\mathrm{J} /(\mathrm{kg} \mathrm{K})) \\
\text { thermal conductivity }\end{array}\end{array}$ & 1005 & 1500 & 840 & 2100 & 384.6 & 1923 \\
$(\mathrm{~W} /(\mathrm{m} \mathrm{k}))$ & 0.0256 & 0.209 & 0.76 & 0.15 & 395 & 0.2 \\
\hline
\end{tabular}

More recently Vince and Dubois [4] examined the dynamics of a long $(L=60 \mathrm{~cm})$ resistive wire placed nearby under the free surface of a silicon oil fluid. The experimental set-up in this experiment allowed them to obtain spatio-temporal dynamics in this quasi-1D configuration. Nice propagative patterns are observed, with some sources or sinks, a kind of defects which are currently under study $[5,6]$. However, the physical mechanism responsible for this experimental observation is not completely understood.

A common feature in the experimental set-up of those experiments is the small heat capacity of the heating device used to destabilize the fluid. In the present paper we present as well some results on quasi-1D convection in a liquid layer with a free surface, but using a copper line heater. Therefore, the main difference with previous studies is that a possible feedback mechanism between convective motions and the heater is reduced. As a consequence, our configuration is closer to the $1 \mathrm{D}$ version of the usual Bénard-Marangoni convection than previous experiments.

A cross-section of the convective cell is shown in fig. 1 . The cell has $4 \mathrm{~cm}$ width and $20 \mathrm{~cm}$ length and is filled with a fluid in contact with the atmosphere (at constant temperature). The lateral walls are made of plexiglas. First surface mirrors (with a low thermal conductivity) are placed in the bottom. A rubber joint is placed between the copper block and the mirrors. The heating device consists of a copper parallelogram $4 \mathrm{~cm}$ wide, $1.5 \mathrm{~cm}$ high and $23 \mathrm{~cm}$ long, with a vertical lame (the heater) $1 \mathrm{~mm}$ wide, $20 \mathrm{~cm}$ long and $5 \mathrm{~mm}$ high. The copper block is drilled with two channels parallel to the heater. A thermostated $( \pm 0.05 \mathrm{~K})$ water circulation of $10 \mathrm{lmin}^{-1}$ keeps the temperature constant. This renders a very uniform temperature on the line heater in contact with the fluid. The heat from the copper block leads to a temperature distribution on the bottom and on the sidewalls. (The conductivities of the different materials of the convective cell are given in table I.) This temperature distribution does not induce qualitative changes in the selected range of temperatures because the main

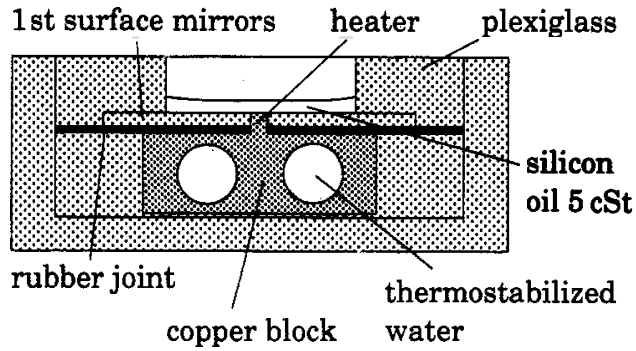

Fig. 1.

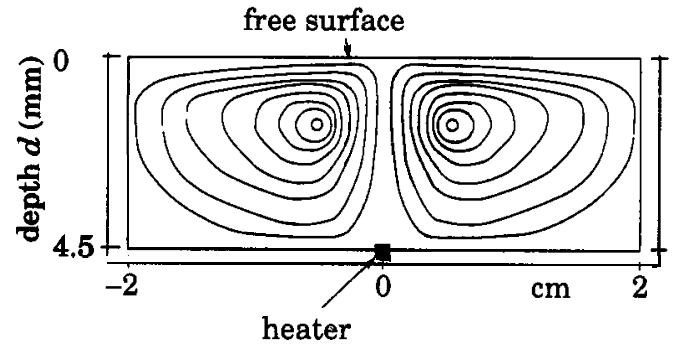

Fig. 2.

Fig. 1. - Sketch of the convective cell.

Fig. 2. - Streamlines for a liquid depth $d=4 \mathrm{~mm}$. Typical velocity at the surface is $5.5 \mathrm{~mm} \mathrm{~s}^{-1}$. 
destabilizing effects are produced by the heater in contact with the fluid. We performed numerical simulations of the conductive state in this configuration and the temperature profiles between the heater and the lateral walls are very similar to a sharp peak centred on the heater. Moreover, when convection appears the width of this temperature distribution peak decreases. The first surface mirrors are placed at the same height as the heater, thus forming a plane bottom $( \pm 10 \mu \mathrm{m})$.

This vessel contains a thin layer $((1 \div 5) \mathrm{mm})$ of $5 \mathrm{cSt}$ silicon oil. (Its properties can be found in ref. [7].) The aspect ratio in these experiments (one-half of the channel width divided by the liquid depth $d$ ) is kept within the range $\Gamma=4 \div 20$.

The visualization technique is a usual shadowgraph by reflection on the bottom mirrors. We restricted the images to the $17 \mathrm{~cm}$ central part of the channel, where meniscus effects do not perturb the measurements. The evolution of the patterns is registered with a CCD camera having a resolution of $512 \times 512$ pixels and 256 grey levels. These images are sent to a computer for image processing.

Experimental results are grouped in two parts: the primary instability and secondary patterns. The primary instability consists in the formation of two rolls parallel to the heater line. They fill the entire width of the container (zero wave number) and have uprising motions over the heater and descending motions near the lateral walls. The appearance of these convective motions is easily observed because they lead to a curvature (concave or convex) of the free interface just above the heating line, thus giving a broader shadow of the (non-reflective) heater. The primary cells appear as soon as a small temperature difference in the linear heater is applied. (Due to the accuracy of our experimental set-up we are not able to determine the corresponding temperature threshold $\Delta T$.) These primary cells are similar to those observed in the lateral heating convection [7].

The physical mechanism of instability is the same as in ref. [1], e.g. a combination of buoyancy and thermocapillary effects. The two dimensionless numbers that correspond to these effects are the Rayleigh number $R$ and the Marangoni number $M$. Typical values for convective thresholds are $R \simeq 670$ and $M \simeq 80$. Then in our experiment the primary instability can appear for $\Delta T<1 \mathrm{~K}$ (for $d>1 \mathrm{~mm}$ ), i.e. smaller than the precision of the temperature measurement.

The streamlines can be observed by adding aluminum powder to the silicon oil, in a quantity small enough to leave the main fluid properties unchanged. (This mixture of liquid and aluminum powder is only used to characterize the primary rolls.) A laser sheet parallel to the bottom plate crosses the convective cell, allowing to visualize the trajectories and the

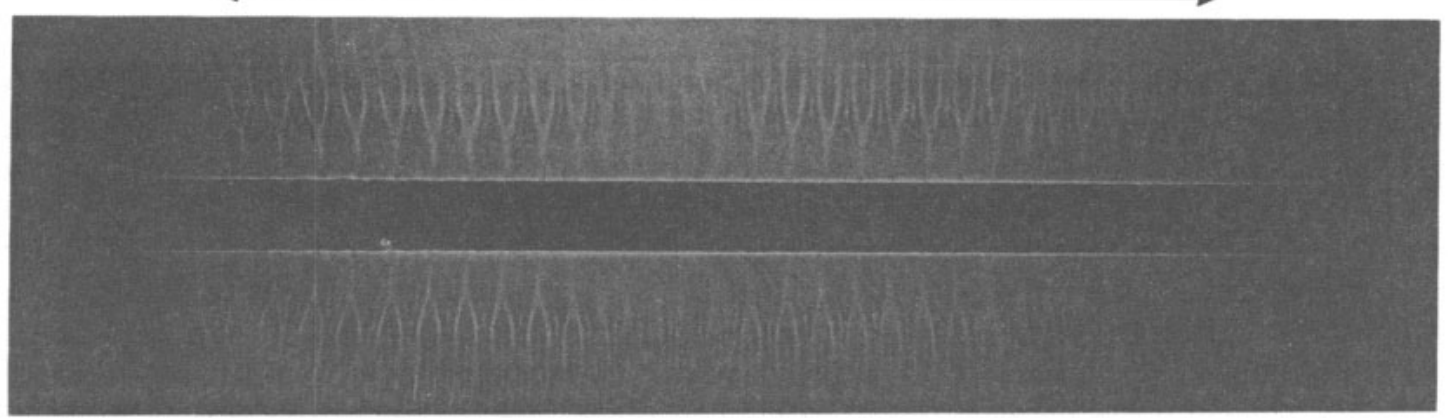

Fig. 3. - Pattern formed by the secondary instability. The central part of the cell is shown. By the effect of surface curvature (meniscus) the vertical and horizontal scales are not the same. Actual vertical height: $2 \mathrm{~cm} .\left(d=3.35 \mathrm{~mm}\right.$ and $\Delta T \simeq 25^{\circ} \mathrm{C}$. 


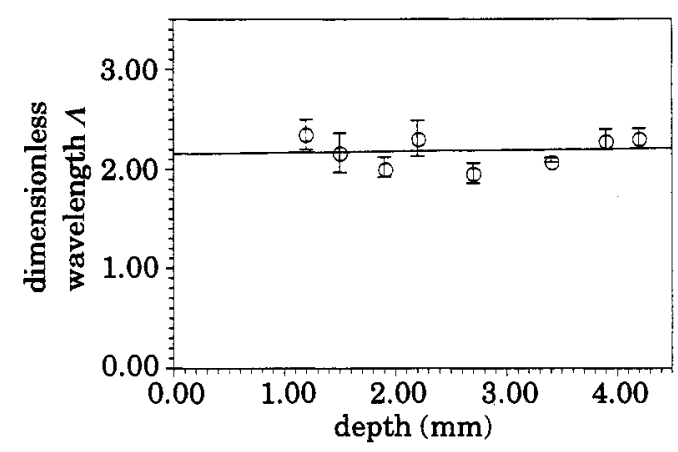

Fig. 4. - Dimensionless wavelength $A$ vs. liquid depth $d$ at $T=50^{\circ} \mathrm{C}$.

velocity of aluminum particles at different heights. The streamlines have the form sketched in fig. 2. The fastest particles, i.e. those travelling near the centre of the cell and the top of the fluid, have velocities between $5 \mathrm{~mm} \mathrm{~s}^{-1}$ and $5.5 \mathrm{~mm} \mathrm{~s}^{-1}$. (The layer was $4.5 \mathrm{~mm}$ deep and the temperature difference about $\Delta T \simeq 20 \mathrm{~K}$.) The velocity distribution is quite asymmetric. This fact is not surprising because the heat supply is very localized. For example, both stagnation points are in the upper part of the cell near the centre (fig. 2).

When the temperature of the heating line is increased, a secondary instability appears. This forms perturbations overimposed on the primary rolls and perpendicular to the linear heater. The temperature difference threshold $\Delta T_{\mathfrak{c}}$ for this instability changes with $d$. By changing $d$ from $1 \mathrm{~mm}$ to $2 \mathrm{~mm}$ the threshold $\Delta T_{\mathrm{c}}$ decreases from $15 \mathrm{~K}$ to $5 \mathrm{~K}$. For a depth range $d=(2.5 \div 4) \mathrm{mm}, \Delta T_{\mathrm{c}}$ remains approximately constant $(\simeq 3 \mathrm{~K})$. For $d>4 \mathrm{~mm}, \Delta T_{\mathrm{c}}$ increases with $d$. (For example, $\Delta T_{\mathrm{c}}=5 \mathrm{~K}$ for $d=5 \mathrm{~mm}$.) (Primary rolls hinder to accurately determine these thresholds.) These thresholds correspond to dimensionless numbers $R \geqslant 3000$ and $M \geqslant 3000$. The transition seems to be gradual and the intensity of the secondary pattern increases with $\Delta T$. An example of a completely developed secondary pattern is given in fig. 3 . $\left(\Delta T \simeq 25^{\circ} \mathrm{C}, d=3.35 \mathrm{~mm}\right.$.)

With the shadowgraph technique we are unable to determine the mechanism responsible for this secondary instability. The pattern could be formed either by quite small convective rolls or by some undulations of the free surface. The existence of a temperature threshold suggests a destabilization of the thermal boundary layer similar to the bimodal instability [8] in Rayleigh-Bénard convection, that would lead to rolls. However, aluminum particle trajectories do not reveal noticeable transverse velocity, therefore suggesting a surface deformation mechanism.

These transverse motions have a well-defined wavelength $\lambda$ and are carried along by the primary rolls. One can also notice in fig. 3 that these transverse convective motions suffer a splitting process when they are carried downstream. The dimensionless wavelengths $\Lambda=\lambda / d$ in these patterns have been measured. We notice that the $\Lambda$ of this pattern $(\Lambda=2.2)$ is more similar to that of the Rayleigh convective rolls $(\Lambda=2.3)$ than to that for Bénard-Marangoni cells $(\Lambda=3.1)$. For a fixed $d, \Lambda$ increases with $\Delta T$. For a fixed $\Delta T, \Lambda$ remains constant when $d$ is changed (see fig. 4).

The dynamical evolution of the secondary transverse pattern can be easily seen by recording a line of pixels parallel to the heating line, quite close to the heater's shadow in fig. 3 . By stacking these lines at regular time intervals one can build a spatio-temporal representation of the evolution of the pattern. A typical stationary pattern is shown in fig. 5 . (Oscillations of small temporal scale are due to the splitting process downstream.) The 
pattern remains stable for more than $4000 \mathrm{~s}$ and no global drift is noticeable in the spatio-temporal diagram.

During the measurements on the secondary instability we realized that the levelling of the convective cell has important consequences on the pattern. Small inclinations (few milliradians, i.e. liquid depth variation of few $\mu \mathrm{m}$ ) in the direction of the linear heater are enough to induce the transverse pattern to propagate in the direction of the heating line (see fig. 6). The pattern travels in the cell from the deep end to the shallow end at a constant (average) velocity. Due to the depth differences, the secondary pattern readjusts its wavelength as it travels (fig. 6). The right side of fig. 6 corresponds to the wall on the shallow end of the container that acts as a sink of waves. (Correspondingly, the deep end is a source of waves.)

The velocity of the travelling patterns for different tilting angles $\theta$ and different depths has been measured. The results are gathered in fig. 7. The mean $\Lambda$ turns out to be independent of $\theta$ as is shown in fig. 8. Similar results are obtained by changing the sign of $\theta$. Therefore, this propagation is not due to imperfections in the cell.

These features of transverse motions can be gathered together in a minimal-phase phenomenological model, following the lines suggested by Brand [9]. First of all, the tilting in the cell breaks the symmetry $x \rightarrow-x$, and is responsible for the drift and for a spatial change

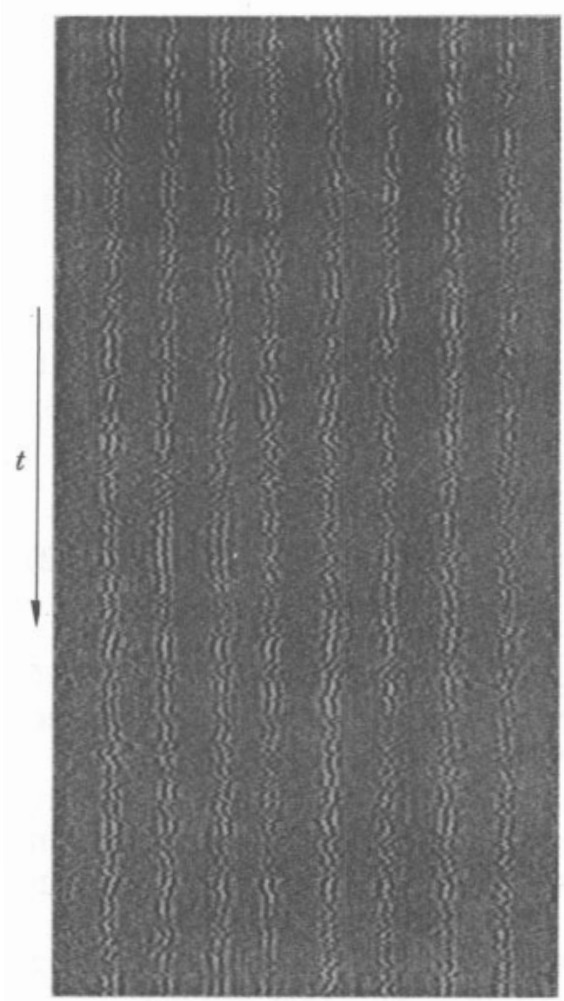

Fig. 5.

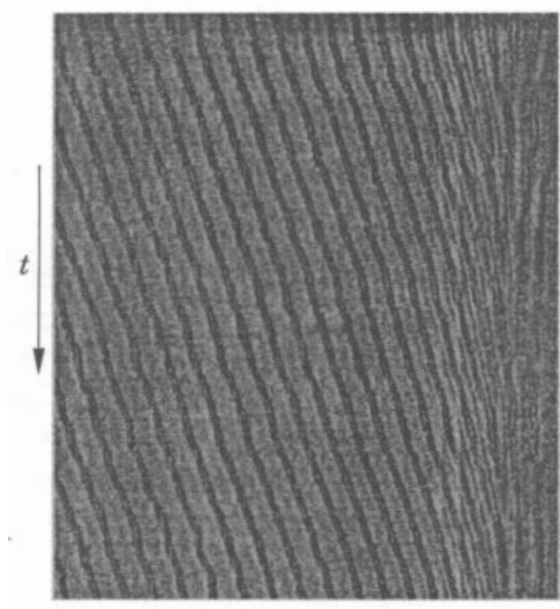

Fig. 6 .

Fig. 5. - Space-time representation of the evolution of the stationary pattern. This image covers $512 \times 256$ pixels $(512 \mathrm{~s} \times 6 \mathrm{~cm})$.

Fig. 6. - Space-time representation of a pattern travelling due to a small tilt $(\theta=2.5 \mathrm{mrad})$. This images covers $373 \mathrm{~s} \times 10 \mathrm{~cm}$. 


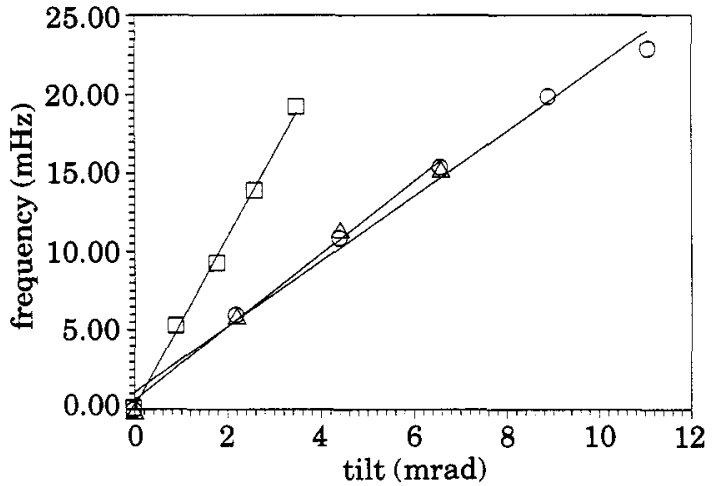

Fig. 7.

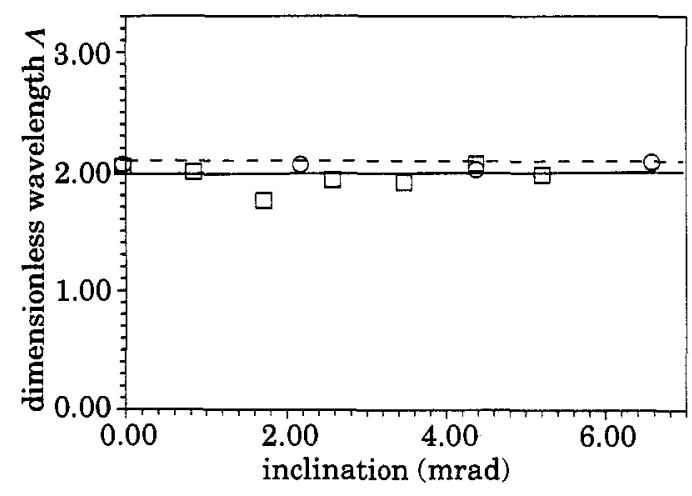

Fig. 8.

Fig. 7. - Frequency of the drifting pattern vs. tilting angle: $\square d=2.7 \mathrm{~mm}, \Delta T=25^{\circ} \mathrm{C} ; \circ d=2.9 \mathrm{~mm}$, $\Delta T=20^{\circ} \mathrm{C} ; \Delta d=3.4 \mathrm{~mm}, \Delta T=25^{\circ} \mathrm{C}$.

Fig. 8. - The dimensionless wavelength $\Lambda$ vs. tilt angle $\theta$, at $T_{\text {heater }}=50^{\circ} \mathrm{C} . \square d=2.7 \mathrm{~mm}$, o $d=3.4 \mathrm{~mm}$.

in the wave number. Then one can take the following phase equation [10]:

$$
\frac{\partial \phi}{\partial t}=\frac{\partial}{\partial x}\left(D(x) \frac{\partial \phi}{\partial x}\right)+\alpha \theta
$$

where $x$ is in the direction of the heating line and the last term $\alpha \theta$ accounts for the phase change due to the inclination. Moreover, we assume an asymmetric diffusion constant that, for small tilting, can be developed as $D(x)=D_{0}+c \theta x$, where $c$ is a constant. (Notice that for $\theta=0$ we recover the usual phase diffusion equation [11].) Then eq. (1) leads to

$$
\frac{\partial \phi}{\partial t}=c \theta \frac{\partial \phi}{\partial x}+D_{0} \frac{\partial^{2} \phi}{\partial x^{2}}+\alpha \theta .
$$

The first two terms account for the propagation at a constant velocity, proportional to $\theta$ (fig. 8). The last two terms lead to a slight variation in the wave number $k \simeq \partial \phi / \partial x$ along the pattern $\left(\Delta k=\left(\alpha / D_{0}\right) \theta \Delta x \propto \Delta d\right)$ which results from a variation in the depth.

We also notice that for a flat layer the secondary stationary pattern is spontaneously destabilized when $\Delta T$ is further increased. It begins to travel to the right or to the left in different regions of the container and some defects (sources or sinks) appear. The description and characterization of these travelling patterns will be reported in a future work.

In conclusion, some preliminary results on the instabilities in a quasi-1D heating convective cell are presented. A primary instability forms a pair of rolls that are parallel to the heater and fill the cell. A secondary instability appears for a further supercritical heating. Further studies using local probes would allow to determine whether these secondary motions are due to surface distortions or to some thermal boundary layer destabilization. Finally, we notice that small tilts in the cell lead to a drift of the secondary pattern with a velocity proportional to the tilting angle. This feature can be partially understood by means of a phenomenological phase equation. 
We are grateful to D. MazA-OzcoIdi, Y. POMEAU, J. E. WESFreid and G. R. Homsy for comments and discussions. This work has been partially supported by DGICYT (Spanish Government) through contract OP90-0098 and PB91-0627 and by Gobierno de Navarra (OF 725/91). One of us (HLM) also acknowledges the DGICYT for a sabbatical grant.

\section{REFERENCES}

[1] KAYSER W. and Berg J., J. Fluid Mech., 57 (1973) 739.

[2] Anthore R., Flament P., Gouesbet P., Rhazi M. and Weill M. E., Appl. Opt., 21 (1982) 2.

[3] Weill M. E., Rhazi M. and Gouesbet G., J. Phys. (Paris), 46 (1985) 1501.

[4] VINCE M. and DuBors M., Europhys. Lett., 20 (1992) 505.

[5] Dubois M., Daviaud F., Ronsin O. and Bergé P., Travelling waves in pure fluids locally heated along wires, in New Trends in Nonlinear Dynamics: Non-Variational Aspects, edited by C. PÉREZ-García, Physica $D, 61$ (1992) 140.

[6] Coullet P., Frisch T. and Plaza F., Sources and sinks of waves patterns, preprint INLN 92.13 (1992).

[7] Ezersky A., Garcimartín A., Burguete J., Mancini H. and Pérez-García C., Phys. Rev. E, 47 (1993) 1126.

[8] Busse F., Rep. Prog. Phys., 41 (1978) 1929.

[9] Brand H. R., Phase dynamics-A review and perspectives, in Propagation in Systems Far from Equilibrium, edited by J. E. WESFREID et al. (Springer, Berlin) 1988, p. 206.

[10] POMEAU Y., private communication.

[11] Pomeau Y. and Manneville P., J. Phys. Lett., 40 (1979) 609. 